\title{
miR-10b promotes cell invasion through RhoC-AKT signaling pathway by targeting HOXD10 in gastric cancer
}

\author{
ZHUO LIU, JIAMING ZHU, HONG CAO, HUI REN and XUEDONG FANG \\ Department of General Surgery, The Second Hospital of Jilin University, Changchun 130041, P.R. China
}

Received October 16, 2011; Accepted December 9, 2011

DOI: $10.3892 / \mathrm{ijo} .2012 .1342$

\begin{abstract}
MicroRNAs play critical roles in tumorigenesis as either oncogenes or tumor suppressors. As a microRNA induced by Twist, miR-10b function as a metastasis driver in different types of cancer, in which the downstream target gene HOXD10 is the main mediator. In gastric tumor species, miR-10b levels were dramatically elevated in lymphoma node metastasis-positive tumor tissues compared with lymphoma node metastasis-free tumor tissues, and were correlated to dowregulation of HOXD10 expression. In gastric cell lines with distinct degrees of differentiation, miR-10b was highly expressed in the cell line with strong metastatic ability. In MNK45 cells, inhibition of miR-10b led to abrogation of cell invasion. While in GES-1 cells, miR-10 overexpression resulted in enhancement of invasiveness through translational inhibition of HOXD10, and constitutive expression of HOXD10 reversed the effects of miR-10b on cell invasion. Furthermore, either knockdown of RhoC or inhibition of AKT activation interfered miR-10-induced invasiveness in GES-1 cells. In summary, these observations suggest that miR-10b can stimulate the upregulation of RhoC and AKT phosphorylation through targeting HOXD10, thus promoting cell invasion in gastric tumors.
\end{abstract}

\section{Introduction}

Gastric cancer is one of the main causes of cancer-associated death in the world (1). Although the incidence of gastric cancer is declining in recent years due to the improved quality of life, its mortality rate is not optimistic (2-4). Malignant gastric cancer cells often possess capabilities of rapid progression and invasion, which contribute to the tumor metastasis and are responsible for the resistance of gastric cancer to the clinical chemotherapeutic strategies and the poor prognosis $(2,5)$.

Correspondence to: Dr Xuedong Fang or Dr Hui Ren, Department of General Surgery, The Second Hospital of Jilin University, Changchun 130041, P.R. China

E-mail: bioscience2011@126.com

E-mail: promoter1998@163.com

Key words: microRNA, signaling pathway, HOXD10, gastric cancer
As one class of the conserved non-coding genes, microRNAs are involved in the pathway of RNA interference, can result in either target mRNAs degradation or block of protein translation (6). It has been known that microRNAs were implicated in diverse physiological process including cell survival, development, and metabolism (7-10). However, in recent years, more and more evidence suggests that dysregulated expression of microRNAs is correlated to tumorigenesis (11). As the late stage of tumor development, metastasis is a complicated process which consists of multiple steps (12). A large amount of studies indicated that microRNAs participated in the regulation of metastasis in different types of cancer (13-15). In gastric cancer, several types of microRNAs were found to play critical roles in tumor metastasis and suggested as potential prognostic biomarkers (16-19).

miR-10b is a Twist-induced microRNA, which was first identified to promote tumor metastasis in breast cancer (15). Upregulated levels of miR-10b was found in metastatic breast cancer cells and miR-10b overexpression robustly increased motility and invasiveness of non-metastatic breast cells (15). Besides breast cancer, aberrant expression pattern of miR-10b was also largely reported in hepatocellular carcinoma, and higher grade glioma, compared to benign tumor tissues $(14,20)$.

HOXD10 identified as the target gene of miR-10b by using computational methods, was closely related to suppression of cell migration and invasion (15). HOXD10 belongs to the HOX genes family, whose expression level was found to be lost during the malignant progression of breast cancer $(15,21)$. The conserved 3'UTR element of HOXD10-encoded mRNA is partially complementary to miR-10b, by which miR-10b abrogates the function of HOXD10 through inhibiting the protein translation but not mRNA degradation (15).

RhoC is a Ras superfamily of GTP-binding protein (22). As one of repression target genes of HOXD10, RhoC was reported to promote tumor metastasis in distinct carcinomas by stimulating the activity of series of kinases including AKT, and mitogenactivated protein kinase (23-25). It was reported that RhoC was specifically up-regulated in gastric cancer with metastatic capability (26), and knockdown of RhoC interfered invasion of gastric cancer cells due to the diminished AKT signaling (27). AKT mediates various basic cellular processes such as cell antiapoptosis, cell cycle (28). A great number of studies revealed abnormal AKT signaling was associated with tumor metastasis (29,30). 
Although a few studies reported the potential relationship between microRNA-10b and occurrence of gastric cancer $(19,31)$, the effect of miR-10b on the metastasis in gastric cancer and the related molecular mechanism remains elusive. Here, we found miR-10b levels were elevated in metastasis-positive gastric tumor species compared with the metastasis-free tumor tissues, which were accompanied by the downregulated expression of HOXD10. In addition, expression of miR-10b was found to be higher in poorly differentiated gastric cell lines, in comparison with well-differentiated cell lines. In gastric cells, we found the ability of cell invasion was enhanced by miR-10b overexpression, while repressed by block of miR-10b activity. Furthermore, we found miR-10b overexpression in gastric cells led to decrease of protein levels of HOXD10, with increase of RhoC expression and AKT phosphorylation, all of which were critical to miR-10b-induced cell invasion.

\section{Materials and methods}

Tumor samples. Patient samples of primary gastric carcinoma were consecutively ascertained between 2007 and 2010. All carcinoma samples were obtained at the time of operation. The human materials were used in accordance with the policies of the institutional review board at the Second Hospital of Jilin University, China.

Cell lines and the plasmids. The GES-1, AGS, N87, and SGC-7901, MKN-45, BGC-823 cell lines were from American Type Culture Collection (ATCC) and cultured under conditions provided by the manufacturer. mir-10b gene was obtained from normal genomic DNA by PCR, then subcloned into a green fluorescent protein (GFP)-expressing, murine stem-cell retrovirus (MSCV)-derived vector (Clontech, Mountain View, CA, USA). The production of retrovirus and infection of target cells were described previously (32). The HOXD10 3'UTR sequence with the binding site for miR-10b was cloned into the pMIRREPORT luciferase construct (7) (Ambion, Austin, TX). The mutant construct of HOXD10 3'UTR was generated by using QuikChange Site-Directed Mutagenesis Kit (Stratagene, La Jolla, CA, USA). For RhoC siRNA construct, the targeting sequence TATATTGCGGACATTGAG (25) was cloned to the vector pGensil-1. HOXD10-pSG5 (addgene) was used for HOXD10 ectopic expression.

Immunoblotting and luciferase reporter assay. For immunoblotting, cells were harvested in RIPA lysis buffer $[150 \mathrm{mM} \mathrm{NaCl}$, $10 \mathrm{mM}$ Tris, $\mathrm{pH} 7.5,1 \%$ NP40, $1 \%$ deoxycholate, $0.1 \%$ SDS, protease inhibitor cocktail (Roche)]. Equal amounts of protein sample was separated by $10 \%$ SDS-PAGE and transferred to PVDF membrane (Millipore, Billerica, MA, USA), the following antibodies were used for immunoblotting: anti-HOXD10 (1:200, Santa Cruz, USA), anti-RHOC (1:200, Santa Cruz), anti- $\beta$ actin (1:10,000, Abcam), anti-Akt (1:200, BD Transduction Laboratories, San Jose, CA, USA); anti-phospho-Akt (ser-473, 1:200, Cell Signaling Technology, Beverly, MA, USA).

For luciferase reporter assay, HEK293T cells were seeded in 24-well plate at density of $3 \times 10^{4}$ cells/well. After $24 \mathrm{~h}$, HEK293T cells were transfected with $200 \mathrm{ng}$ firefly luciferase reporter gene construct (per well) and $1 \mathrm{ng}$ pRL-SV40 Renilla luciferase constructs (per well) for normalization were cotrans- fected. Twenty-four hours post-transfection cells were collected and luciferase activity was measured with the Dual-Luciferase Reporter Assay System (Promega, Madison, WI, USA).

miRNA detection and real-time PCR. For the detection of miR-10b, isolation of small RNAs was done using the mirVana miRNA Isolation Kit (Ambion). Detecting the miRNA mature form was performed using the mirVana qRT-PCR miRNA Detection Kit (Ambion). For HOXD10, total RNA from cells was extracted using TRNzol-A ${ }^{+}$(Tiangen, China), and reversetranscribed into cDNA with the Reverse Transcriptase M-MLV (Takara, Otsu, Japan). qRT-PCR was performed using the SYBR Green qPCR Master Mix (Tiangen) on stratagem Mx3000P. U6 small nuclear RNA was used as an internal control. The primers used for detection of miR-10b were: forward probe: TACCCTGTAGAACCGAATTTGTG, reverse probe: universal qPCR primer. The primers used for detection of HOXD1, forward: GACATGGGGACCTATGGAATGC reverse: TGGTGGTTCACTTCTCTTTTGG.

Immunohistochemistry and in situ hybridization. Tumor or matched normal tissues were fixed by paraformaldehyde (4\%). After embedding by paraffin, samples were sectioned. Sections were incubated with HOXD10 antibody (1:200). The bound antibodies were detected with the biotin-streptavidin-peroxidase system (Vector Labs, Burlingame, CA, USA) and 3,3'-diaminobenzidine hydrochloride (DAB, Sigma, St. Louis, MO, USA) was used as chromogen. In situ hybridization was performed using DIG-labeled locked nucleic acid (LNA)-based probe specific for miRNA-10b (Exiqon, Vedbaek, Denmark). Detection of the probe was carried out using digoxigenin antibody $(21 \mathrm{H} 8 ; 1: 200$, ab420, Abcam Incorporated, Cambridge, MA, USA), LSAB2 System-HRP (Dako, Carpinteria, CA, USA) and liquid DAB ${ }^{+}$ Substrate Chromogen System (Dako) according to the manufacturer's instructions.

In vitro migration and invasion assay. For invasion assays, matrigel was diluted by medium without serum or growth factors for $1 \mathrm{mg} / \mathrm{ml}$, then coated on the membrane (24-well insert; pore size, $8 \mu \mathrm{m}$; BD Biosciences). Cells were plated in medium for 50,000 per well without serum or growth factors, and medium supplemented with serum or growth factors was used as a chemoattractant in the lower chamber. The cells were incubated for $24 \mathrm{~h}$. Cells that did not migrate or invade were removed by a cotton swab. Cells on the lower surface of the membrane were stained with the Diff-Quick Staining Set (Dade Behring, Alemanha) and counted. For transwell migration assays, $2.5 \times 10^{4}$ cells were plated in the top chamber with the non-coated membrane. The others methods were the same as invasion assays.

Statistical analysis. Data are presented as mean \pm SEM. Student's t-test (two-tailed) was used to compare two groups ( $\mathrm{P}<0.05$ was considered significant) unless otherwise indicated ( $\chi^{2}$ test).

\section{Results}

miR-10b level is elevated in metastasis-positive gastric cancer. To explore the potential role of miR-10b in the gastric cancer development, expression of miR-10b was investigated in tumor 
A

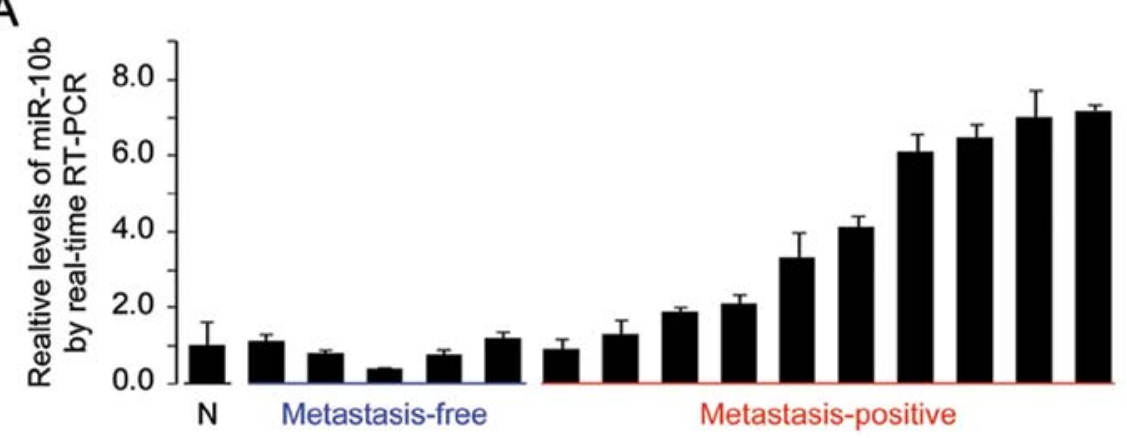

B

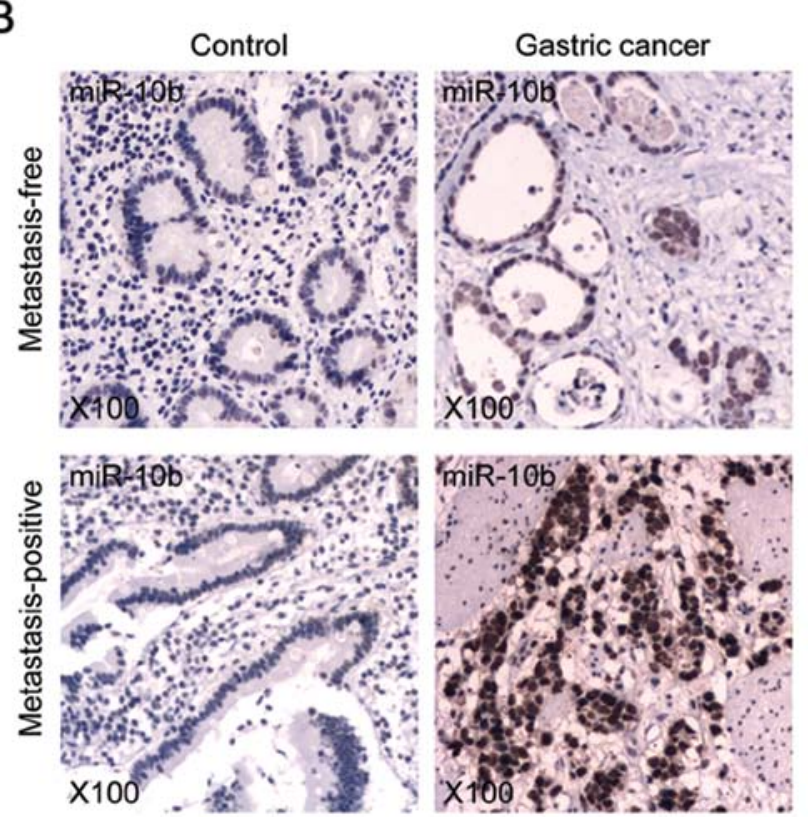

Figure 1. miR-10b expression level is associated with the metastasis outcome in gastric cancer. (A) miR-10b levels in gastric tumors. miR-10b was detected by real-time PCR in 15 primary gastric tumors from patients with indicated status of metastasis. Error bars indicate SEM of triplicate experiments. $\beta$-actin mRNA was used as the internal control. (B) miR-10b levels in gastric tumors. miR-10b were detected in 15 primary gastric tumors by in situ hybridization and immunohistochemistry.

species from 15 gastric cancer patients, which were collected and sorted into two groups shown as species with lymphoma node metastasis-free and species with lymphoma node metastasispositive (Fig. 1). Apparently, results of real-time PCR indicated miR-10b levels in tumor tissues of metastasis-positive was increased 4-8-fold higher than those of metastasis-free tissues and the matched tumor adjacent normal tissues (Fig. 1A). To confirm the real-time PCR data, we performed in situ hybridization to detect the levels of miR-10b. Consistently, we found miR-10b expression was enhanced in metastasis-positive tissues compared with metastasis-free tissues (Fig. 1B). These data revealed miR-10b would be involved in the process of gastric cancer metastasis.

miR-10b is highly expressed in metastatic gastric cancer cells. Data from tumor tissues imply increased miR-10b levels would facilitate metastasis of gastric cancer cells. We next investigated miR-10b expression levels in different gastric cancer cell lines, which were divided into 4 types according to distinct degrees of differentiation (Fig. 2). It should be noted that the differentiation degree is reversely correlated with the capability of metastasis in these gastric cancer cell lines. miR-10b could not be detected in normal gastric cells nor in well-differentiated gastric cancer cells (Fig. 2). The weak expression of miR-10b was observed in cells with moderately differentiation (Fig. 2). However, we found miR-10b was highly expressed in poorly differentiated gastric cancer cell lines, such as BGC-823 and MKN45, which possess strong metastatic ability (Fig. 2). Thus, these results indicated that miR-10b expression level was positively related to metastatic ability of gastric cancer cells.

miR-10b promotes invasion of gastric cancer cells. For further exploring whether miR-10b is indispensable for metastasis of gastric cancer cells, we examined effects of miR-10b inhibition on cell metastasis in MNK45 cells, which express high levels of miR-10b (Fig. 2). As shown in Fig. 3A and B, inhibition of miR-10b activity by miR-10b inhibitor had no obviously effects on migration of MNK45 cells, while severely blocked cell invasion ability. In addition, effects of miR-10b overexpression on migration or invasion of GES-1 cells was investigated. Evidently, the results of real-time PCR indicated miR-10b expression level was substantially elevated after infected with miR-10b-expressing 
A

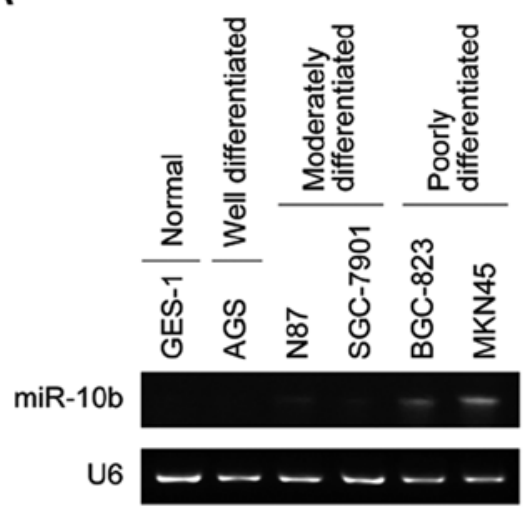

B

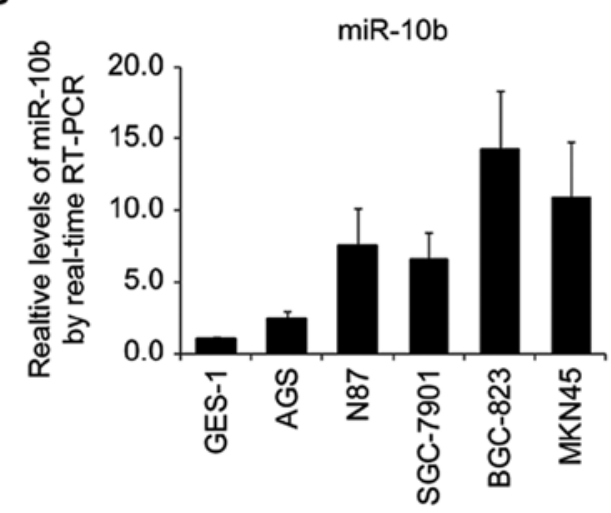

Figure 2. High expression of miR-10b in metastatic gastric cancer cells. (A) miR-10 levels in distinct gastric cancer cell lines. miR-10b was detected by real-time PCR in gastric cancer cell lines of various degrees of differentiation. (B) Quantitation of the relative fluorescence intensities shown in (A). A representative experiment is shown in triplicate along with SEM.

A
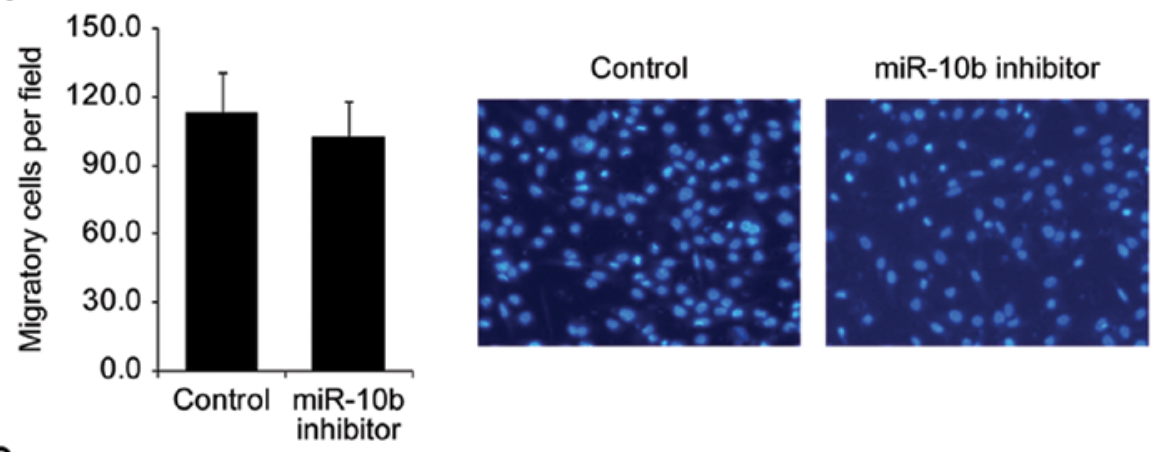

\section{B}
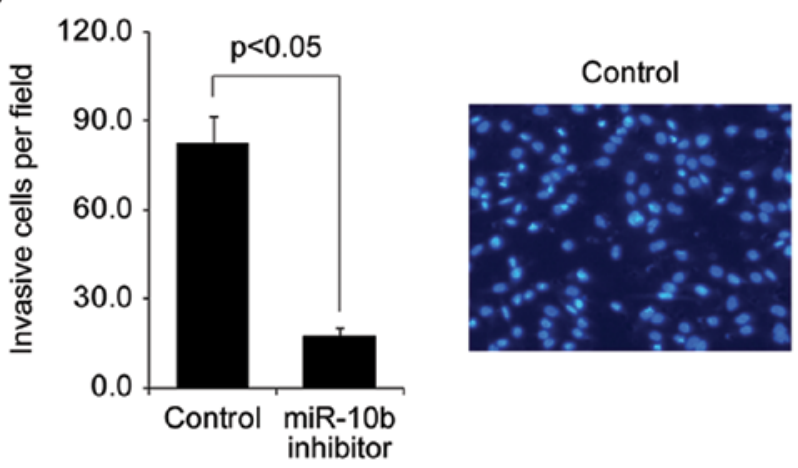

miR-10b inhibitor

C
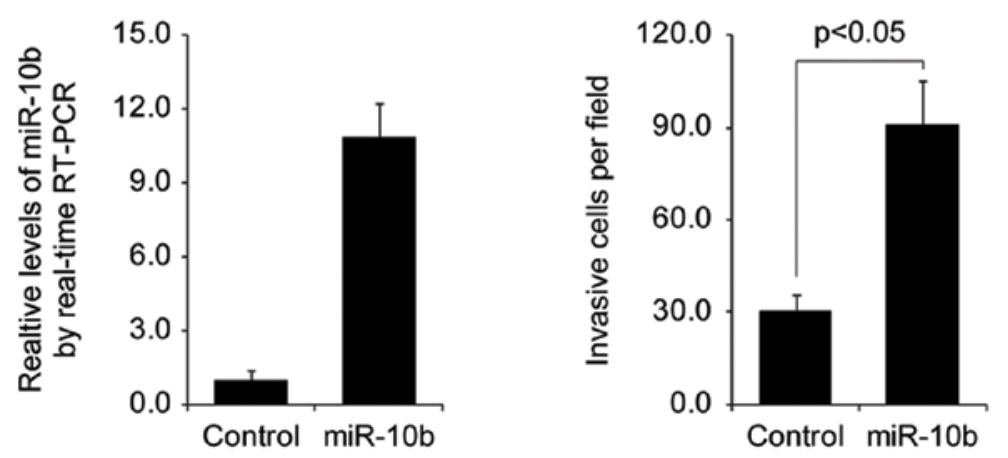

Figure 3. miR-10b positively regulates cell migration and invasion. (A) miR-10b has no effects on migration of MNK45 cells. Cell migratory ability was analyzed by Transwell migration assay. MNK45 cells were treated with or without miR-10b inhibitor (quantified: left). Magnification: right, x200. (B) miR-10b is critical to invasion of MNK 45 cells. Cell invasion ability was analyzed by matrigel invasion assay. MNK45 cells were treated with or without miR-10b inhibitor (quantified: left). Magnification: right, x200. (C) miR-10b promotes invasion of GES-1 cells. GES-1 cells were infected with the retrovirus with miR-10b-expressing or empty vector. Cell invasion ability was analyzed by matrigel invasion assay. A representative experiment is shown in triplicate along with SEM. 
A
5 '...UUUUUUCAUCG - UAAUGCAGGGUAA...
HOXD10 3' UTR
3' UGUUUAAGCCAAGAUGUCCCAU
hsa-miR-10b
$5^{\prime}$
TCGTAATGGTCCCAT HOXD10 3' UTR mutant

B
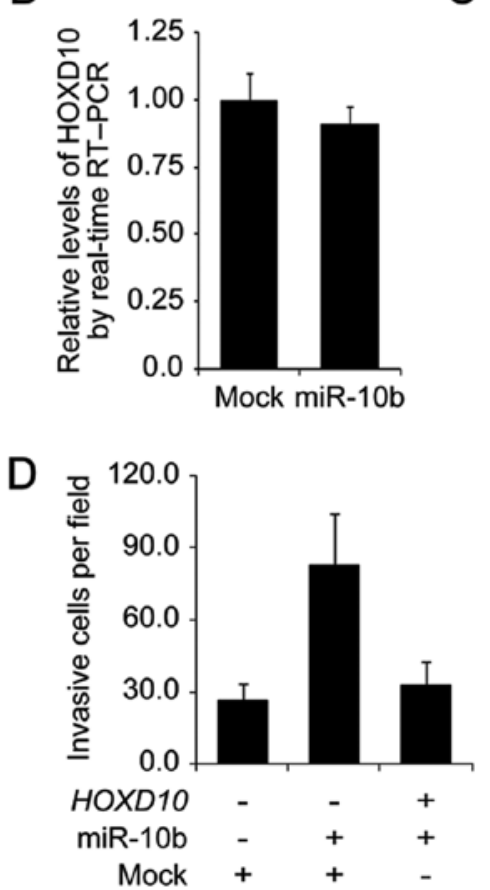

C
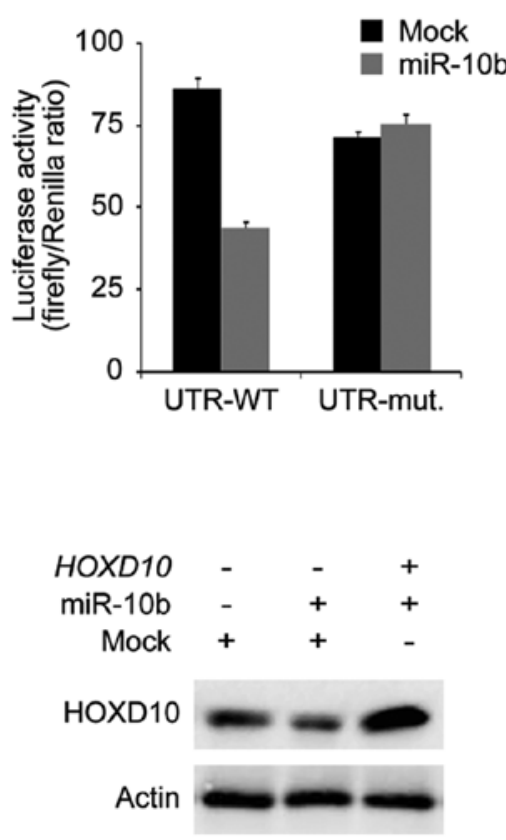

Figure 4. miR-10b promotes cell invasion by targeting HOXD10. (A) Potential target sites for miR-10b in the 3'UTRs of HOXD10 mRNAs. (B) miR-10b cannot alter mRNA level of HOXD10. GES-1 cells were infected with retrovirus with the miR-10b-expressing or empty vector (mock). HOXD10 mRNA level was detected by real-time PCR. Data were normalized to the level of GAPDH mRNA. (C) Luciferase activity of wild-type (WT-UTR) or mutant (mut-UTR). HOXD10 3'UTR reporter gene in GES-1 cells infected with retrovirus with the miR-10b-expressing or mock. (D) miR-10b induces cell invasion by targeting HOXD10. GES-1 cells were infected with retrovirus with or without the miR-10b-expressing and were infected with adenovirus with HOXD10-expressing vector resistant to miR-10b-mediated inhibition. The figure on the left shows cell invasion ability analyzed by matrigel invasion assay. On the right, HOXD10A level was detected by immunoblotting. A representative experiment is shown in triplicate along with SEM.

lentivirus, and miR-10b overexpression largely enhanced cells invasiveness (Fig. 3C). These observations suggest miR-10b selectively regulates invasion under the context of gastric cancer cells.

miR-10b induces cell invasion through targeting HOXD10. A previous study indicated HOXD10 is the main target of miR-10b (15) (Fig. 4A), which led us to examine the role of HOXD10 in miR-10b-induced invasion of gastric cancer cells. In MNK45 cells, miR-10b overexpression induced the decrease of HOXD10 protein level, while could not alter mRNA level of HOXD10 (Fig. 4B), which is consistent with mechanism that miR-10b represses function of HOXD10 through translational inhibition but not mRNA degradation (15). Next, effects of miR-10b on HOXD10 function were examined by using luciferase reporter gene analysis in MNK45 cells. miR-10b overexpression only remarkably reduced luciferase activity of reporter gene with wild-type, but not mutant HOXD10 3'UTR, indicating the essential role of miR-10b binding site within HOXD10 3'UTR (15). We further determined whether repressed HOXD10 levels would be critical to invasiveness induced by the miR-10b over-expression. The construct expressing constitutive HOXD10 lacking 3'UTR of HOXD10-encoding mRNA was overexpressed in MNK45 cells. Evidently, the resistant form of HOXD10 completely interfered with miR-10b-stimulated enhancement of cell invasiveness (Fig. 4D), indicating its important role as the target of miR-10b in this process.

miR-10b induces cell invasion through AKT signaling pathway. As the downstream target gene repressed by HOXD10, RhoC often positively regulate cell metastasis $(15,25)$. Thus, we next determined whether RhoC mediates effects of miR-10b-induced HOXD10 inhibition on invasiveness of gastric cancer cells. In MNK45 cells, overexpressed miR-10b resulted in decrease of HOXD10 protein level, while led to upregulation of RhoC level, suggesting RhoC expression is inhibited by HOXD10 in gastric cell line (Fig. 5A). In addition, knockdown of RhoC by siRNA partially abrogated invasiveness induced by miR-10b overexpression, which indicated RhoC is the important downstream effector of miR-10b in invasion of gastric cancer cells (Fig. 5B). RhoC was reported to drive cancer cell metastasis by stimulating AKT activity $(23,24,27)$. Consistently, we found miR-10b overexpression led to robust phosphorylation of AKT in MNK45 cells, which was completely blocked by knockdown of RhoC 
A

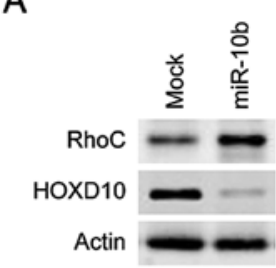

B

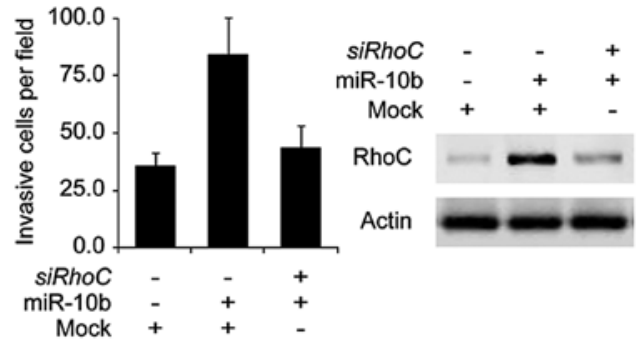

C

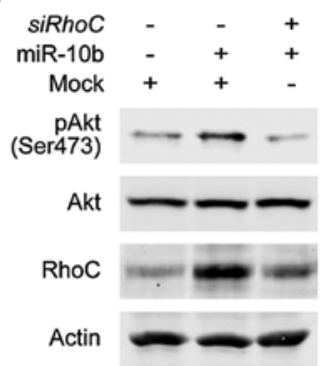

D

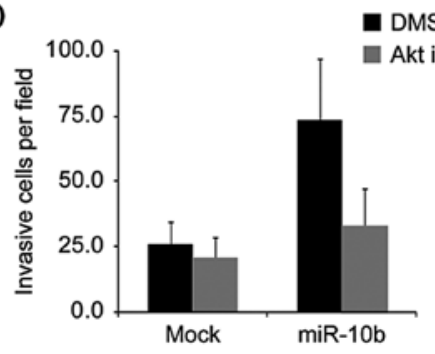

Figure 5. miR-10b promotes cell invasion in the Akt-dependent pathway. (A) miR-10b induces upregulation of RhoC level. GES-1 cells were infected with retrovirus with miR-10b-expressing or control vector. HOXD10 and RhoC were detected by immunoblotting. (B) RhoC was critical to miR-10b-induced cell invasion. GES-1 cells were infected with adenovirus with RhoC-siRNA-expressing or control vector. Left panel, matrigel invasion assay of miR-10b-infected cells, infected with adenovirus with RhoC-siRNA-expressing or control vector. Right panel, immunoblotting of RhoC. (C) miR-10b leads to AKT activation through RhoC. GES-1 cells were infected with adenovirus with RhoC-siRNA-expressing or control vector. pAKT and RhoC were detected by immunoblotting. (D) Inhibition of AKT represses miR-10b-induced cell invasion. GES-1 cells were treated with or without AKT inhibitor III. Cell invasion ability was analyzed by matrigel invasion assay. A representative experiment is shown of triplicate along with SEM.
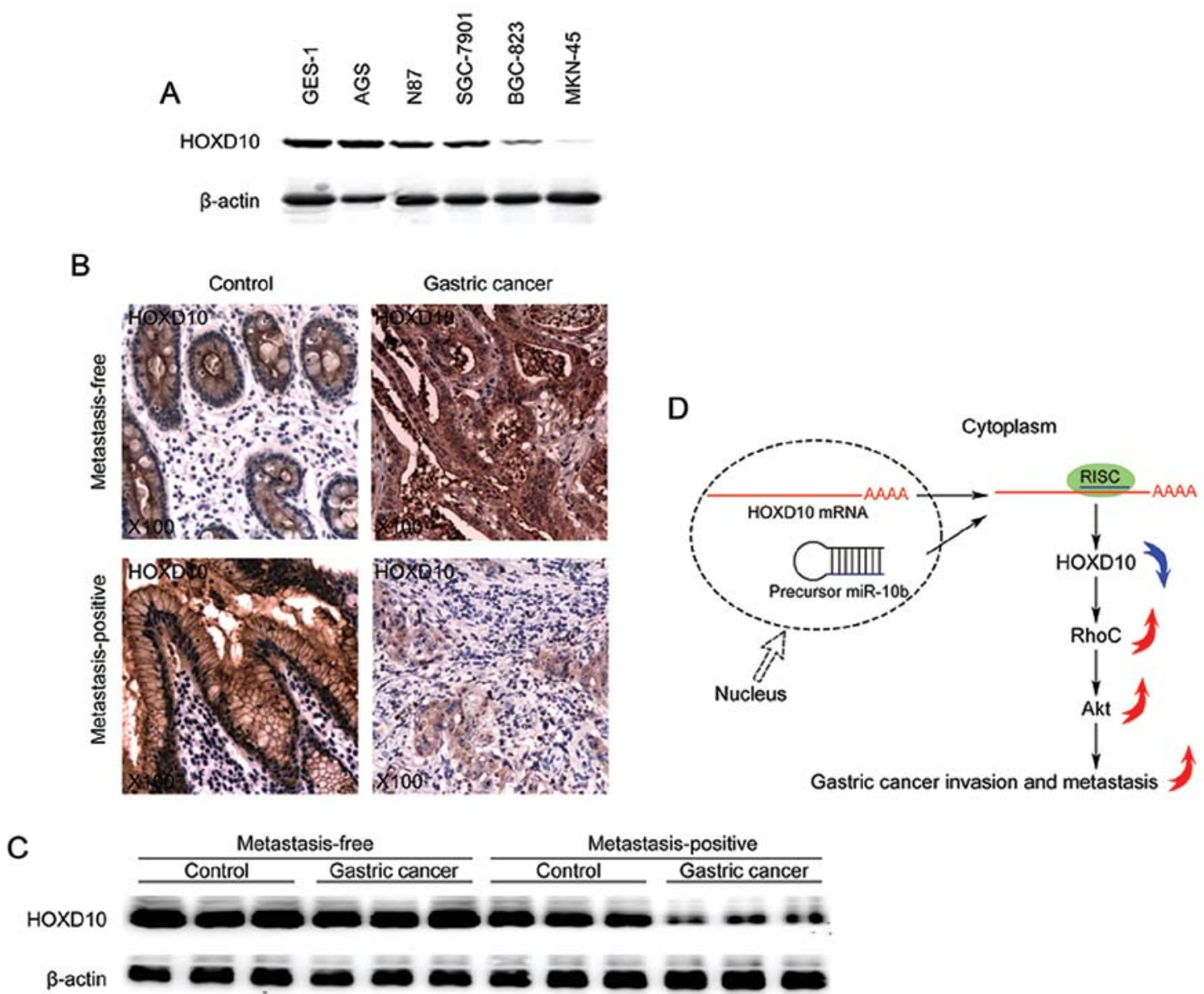

Figure 6. Relationship between HOXD10 levels and gastric cancer metastasis. (A) HOXD10 levels in distinct gastric cancer cell lines. HOXD10 was detected by immunoblotting in gastric cancer cell lines of various degrees of differentiation. (B) HOXD10 levels in gastric tumors. HOXD10 were detected in 15 primary gastric tumors by immunohistochemistry. (C) HOXD10 levels in gastric tumors. HOXD10 were detected in 15 primary gastric tumors by immunoblotting. (D) Models of miR-10b-induced cell invasion in gastric cancer. Through translational inhibition, miR-10b represses HOXD10 function, thereby leads to increase of RhoC expression and activation of AKT. Gastric cancer invasion and metastasis was enhanced by upregulated miR-10b. 
(Fig. 5C). We further examined effect of AKT activation on miR10b-induced invasiveness of MNK45 cells, and found inhibition of AKT activity by AKT inhibitor III partially interfered with cell invasion (Fig. 5D). Hence, RhoC seems to promote invasiveness of gastric cancer cell induced by miR-10b through AKT signaling pathway.

Inverse correlation between HOXD10 levels and metastasis in gastric cancer. As HOXD10 was one of the main targets of miR-10b which plays an important role in tumor metastasis $(15,21)$, the protein levels of HOXD10 were further analyzed in distinct gastric cancer cell lines and gastric cancer species. We found HOXD10 protein levels were evidently decreased in poorly differentiated gastric cancer cells compared with the other cell lines with low metastatic capability (Fig. 6A). In tumor tissues, mRNA and protein levels of HOXD10 were examined by using immunohistochemistry and Western blotting, respectively. We found HOXD10 expression was dramatically downregulated in metastasis-positive tissues (Fig. 6B and C). Thus, changes of HOXD10 expression in cell lines and species of gastric cancer suggest the suppressive role of HOXD10 in in gastric cancer metastasis.

\section{Discussion}

MicroRNAs have been known to be implicated in tumor progression in various types of human cancer including gastric cancer (17-19), which is one of the most common malignant tumors (1). Recently, many studies identified a series of microRNAs which function as either oncogene of tumor suppressor in gastric cancer. miR-10b is the Twist-induced microRNA, reported to be associated with breast tumor metastasis (15). A small number of reports suggested miR-10b might affect development of gastric tumor $(19,31)$, while exact roles of miR-10b in distinct stages of tumor progression and related molecular mechanisms remain elusive. In this study, we found the critical role of miR-10b in gastric cancer cell invasion. Overexpression of miR-10b led to increased RhoC and AKT signaling by targeting HOXD10, which facilitates cell invasion in gastric cancer (Fig. 6D). In human gastric tumor species with tendency to metastasis, miR-10b was highly expressed, accompanied by decreased HOXD10 levels (Figs. 1 and 6B and C). Consistent with previous finding that miR-10b could drive tumor metastasis (15), elevated levels of miR-10b revealed miR-10b would also contribute to metastasis in gastric cancer. As one of gene targets of miR-10b, HOXD10 is considered as the main effector which negatively regulates tumor metastasis $(15,21)$. Thus, downregulation of HOXD10 might result from enhanced miR-10b expression. Further, high levels of miR-10b were detected in gastric cancer cell lines with low degree of differentiation that possess strong ability of metastasis (Fig. 2). In contrast, HOXD10 expression was found to be attenuated in lowly differentiated gastric cancer cell lines compared with those of well differentiation (Fig. 6A). These results suggested miR-10b could reduce the threshold of metastasis in gastric cancer as the positive regulator, which is inversely related to HOXD10.

Expression of miR-10b in tumor tissues and cell lines implied its essential role in metastasis. In MNK45 cells, inhibition of miR-10b abrogated invasiveness (Fig. 3B). On the contrary, overexpressed miR-10b enhanced invasiveness of GES-1
(Fig. 3C). Thus, miR-10b seems to be indispensable for cell invasion and elevation of miR-10b alone is enough to promote cell invasion in the context of gastric cancer. As shown in Fig. 2A, miR-10b levels have no effects on migration of gastric cancer cells, which would be attributed to no participation of miR-10b in the signaling pathway related to cell migration or migration capability, which could not be hindered by blocking miR-10b alone in cancer cells we used.

Previous studies indicated miR-10b restrained HOXD10 by translational inhibition, in which the 3'UTR miR-10b binding site is crucial (15). Likely, in gastric cancer cells, we found miR-10b interfered with translation of HOXD10 but not mRNA level, and the vector expressing mutational HOXD10 3'UTR was resistant to the inhibition (Fig. 4B). The constitutive expression of HOXD10 abrogated enhancement of cell invasion induced by miR-10b overexpression (Fig. 4C and D). These observations indicated miR-10b promoted invasiveness of gastric cancer cells by directly targeting HOXD10.

HOXD10 has been known to repress expression of genes involving in tumor metastasis including RhoC. In GES-1 cells, miR-10b overexpression led to elevation of RhoC (Fig. 5A), and knockdown of RhoC partially abrogated effects of miR-10b overexpression on cell invasion (Fig. 5B), which indicated Rho contributed to miR-10b-induced invasiveness as the target gene of HOXD10. The partial effects of RhoC would be explained by that the other target genes of HOXD10 are also involved in these processes. AKT activation has been found to be responsible for RhoC-mediated metastasis of cancer cells $(23,24,27)$. In GES-1 cells, knockdown of RhoC resulted in attenuation of AKT phosphorylation levels, and inhibition of AKT activation partially blocked miR-10b-induced invasiveness of gastric cancer cell (Fig. 5C and D). Thus, blocking of AKT activity would be useful for preventing metastasis of gastric cancer cells.

In conclusion, our study suggests high levels of miR-10b are associated with the metastatic degree in gastric cancer patients and gastric cancer cell lines. In addition, miR-10b overexpression promoted invasiveness of gastric cells by targeting HOXD10, which partially passed through RhoC-AKT signaling. These observations shed new light on mechanisms underlying invasion of gastric cancer cells and supply novel therapeutic targets in inhibiting metastasis in gastric cancer.

\section{Acknowledgements}

This work was supported by grants from Innovative Team Construction Project of Jilin University and the Science and Technology Development Plan of Jilin Province (20100725).

\section{References}

1. Hohenberger P and Gretschel S: Gastric cancer. Lancet 362: 305-315, 2003.

2. Ohtsu A: Chemotherapy for metastatic gastric cancer: past, present, and future. J Gastroenterol 43: 256-264, 2008.

3. Lordick F, Grenacher L, Röcken C, Ebert M, Moehler M and Schumacher G: Diagnosis and treatment of gastric cancer. Dtsch Med Wochenschr 135: 1671-1682, 2010.

4. Dank M: Recent advances in the treatment of gastric cancer. Magy Onkol 54: 337-341, 2010.

5. Moskvina LV and Mal'kov PG: Current views of the molecular mechanisms of gastric cancer progression. Arkh Patol 72: 58-61, 2010. 
6. Huntzinger E and Izaurralde E: Gene silencing by microRNAs: contributions of translational repression and mRNA decay. Nat Rev Genet 12: 99-110, 2011.

7. Cheng AM, Byrom MW, Shelton J and Ford LP: Antisense inhibition of human miRNAs and indications for an involvement of miRNA in cell growth and apoptosis. Nucleic Acids Res 33: 290-297, 2005

8. Pauli A, Rinn JL and Schier AF: Non-coding RNAs as regulators of embryogenesis. Nat Rev Genet 12: 136-149, 2011.

9. Small EM and Olson EN: Pervasive roles of microRNAs in cardiovascular biology. Nature 469: 336-342, 2011.

10. Chen CZ, Li L, Lodish HF and Bartel DP: MicroRNAs modulate hematopoietic lineage differentiation. Science 303: 83-86, 2004.

11. Erson AE and Petty EM: miRNAs and cancer: new research developments and potential clinical applications. Cancer Biol Ther 8: 2317-2322, 2009.

12. Fidler IJ: The pathogenesis of cancer metastasis: the 'seed and soil’ hypothesis revisited. Nat Rev Cancer 3: 453-458, 2003.

13. Hatziapostolou $M$ and Iliopoulos D: Epigenetic aberrations during oncogenesis. Cell Mol Life Sci 68: 1681-1702, 2011.

14. Ladeiro Y, Couchy G, Balabaud C, Bioulac-Sage P, Pelletier L, Rebouissou S and Zucman-Rossi J: MicroRNA profiling in hepatocellular tumors is associated with clinical features and oncogene/tumor suppressor gene mutations. Hepatology 47: 1955-1963, 2008.

15. Ma L, Teruya-Feldstein J and Weinberg RA: Tumour invasion and metastasis initiated by microRNA-10b in breast cancer. Nature 449: 682-688, 2007.

16. Wang Z, He YL, Cai SR, Zhan WH, Li ZR, Zhu BH, Chen CQ, Ma JP, Chen ZX, Li W and Zhang LJ: Expression and prognostic impact of PRL-3 in lymph node metastasis of gastric cancer: its molecular mechanism was investigated using artificial microRNA interference. Int J Cancer 123: 1439-1447, 2008.

17. Tseng $\mathrm{CW}$, Lin $\mathrm{CC}$, Chen $\mathrm{CN}$, Huang $\mathrm{HC}$ and Juan HF: Integrative network analysis reveals active microRNAs and their functions in gastric cancer. BMC Syst Biol 5: 99, 2011.

18. Wu WK, Lee CW, Cho CH, Fan D, Wu K, Yu J and Sung JJ: MicroRNA dysregulation in gastric cancer: a new player enters the game. Oncogene 29: 5761-5771, 2010.

19. Li X, Zhang Y, Zhang Y, Ding J, Wu K and Fan D: Survival prediction of gastric cancer by a seven-microRNA signature. Gut 59: $579-585,2010$

20. Sasayama T, Nishihara M, Kondoh T, Hosoda K and Kohmura E MicroRNA-10b is overexpressed in malignant glioma and associated with tumor invasive factors, uPAR and RhoC. Int J Cancer 125: 1407-1413, 2009
21. Carrio M, Arderiu G, Myers C and Boudreau NJ: Homeobox D10 induces phenotypic reversion of breast tumor cells in a threedimensional culture model. Cancer Res 65: 7177-7185, 2005.

22. Rose R, Weyand M, Lammers M, Ishizaki T, Ahmadian MR and Wittinghofer A: Structural and mechanistic insights into the interaction between Rho and mammalian Dia. Nature 435: $513-518,2005$.

23. Ruth MC, Xu Y, Maxwell IH, Ahn NG, Norris DA and Shellman YG: RhoC promotes human melanoma invasion in a PI3K/Akt-dependent pathway. J Invest Dermatol 126: 862-868, 2006.

24. Iiizumi M, Bandyopadhyay S, Pai SK, Watabe M, Hirota S, Hosobe S, Tsukada T, Miura K, Saito K, Furuta E, Liu W, Xing F, Okuda H, Kobayashi A and Watabe K: RhoC promotes metastasis via activation of the Pyk2 pathway in prostate cancer. Cancer Res 68: 7613-7620, 2008.

25. Wu M, Wu ZF, Rosenthal DT, Rhee EM and Merajver SD: Characterization of the roles of RHOC and RHOA GTPases in invasion, motility, and matrix adhesion in inflammatory and aggressive breast cancers. Cancer 116: 2768-2782, 2010

26. Liu N, Zhang G, Bi F, Pan Y, Xue Y, Shi Y, Yao L, Zhao L, Zheng $\mathrm{Y}$ and Fan D: RhoC is essential for the metastasis of gastric cancer. J Mol Med 85: 1149-1156, 2007.

27. Sun HW, Tong SL, He J, Wang Q, Zou L, Ma SJ, Tan HY, Luo JF and Wu HX: RhoA and RhoC -siRNA inhibit the proliferation and invasiveness activity of human gastric carcinoma by Rho/ PI3K/Akt pathway. World J Gastroenterol 13: 3517-3522, 2007.

28. Garofalo M, Quintavalle C, Zanca C, De Rienzo A, Romano G, Acunzo M, Puca L, Incoronato M, Croce CM and Condorelli G: Akt regulates drug-induced cell death through Bcl-w downregulation. PLoS One 3: e4070, 2008.

29. Sheng S, Qiao M and Pardee AB: Metastasis and AKT activation. J Cell Physiol 218: 451-454, 2009.

30. Jiang BH and Liu LZ: PI3K/PTEN signaling in tumorigenesis and angiogenesis. Biochim Biophys Acta 1784: 150-158, 2008.

31. Kim K, Lee HC, Park JL, Kim M, Kim SY, Noh SM, Song KS Kim JC and Kim YS: Epigenetic regulation of microRNA-10b and targeting of oncogenic MAPRE1 in gastric cancer. Epigenetics 6: 740-751, 2011.

32. Stewart SA, Dykxhoorn DM, Palliser D, Mizuno H, Yu EY, An DS, Sabatini DM, Chen IS, Hahn WC, Sharp PA, Weinberg RA and Novina CD: Lentivirus-delivered stable gene silencing by RNAi in primary cells. RNA 9: 493-501, 2003. 